\title{
Commute Equilibrium for Mixed Networks with Autonomous Vehicles and Traditional Vehicles
}

\author{
Yangbeibei Ji, ${ }^{1}$ Mingwei Xu, ${ }^{1}$ Hua Wang, ${ }^{2}$ and Chaowu $\operatorname{Tan}^{3}$ \\ ${ }^{1}$ School of Management, Shanghai University, 99 Shangda Road, Shanghai 200444, China \\ ${ }^{2}$ School of Economics and Management, Tongji University, 1239 Siping Road, Shanghai 200092, China \\ ${ }^{3}$ College of Transport \& Communications, Shanghai Maritime University, 1550 Haigang Road, Pudong, Shanghai 201306, China \\ Correspondence should be addressed to Yangbeibei Ji; jybb@shu.edu.cn
}

Received 9 May 2017; Revised 21 September 2017; Accepted 18 October 2017; Published 12 November 2017

Academic Editor: Xiaobo Qu

Copyright (C) 2017 Yangbeibei Ji et al. This is an open access article distributed under the Creative Commons Attribution License, which permits unrestricted use, distribution, and reproduction in any medium, provided the original work is properly cited.

Recent development of autonomous vehicle (AV) provides new travel opportunities for citizens, and traditional vehicles (TVs) will still be used for a long time. Therefore, it is highly possible that both AVs and TVs will be used as travel modes in a city. In a transportation system with both AVs and TVs, the traffic pattern is worthy of studying. This paper investigates user equilibrium traffic pattern based on the traditional bottleneck model considering AVs and TVs. For both TVs and AVs, travel costs include queuing delay and schedule delay. However, they also have different components of travel costs; more specifically, for AVs, passengers have to pay a riding fare, and, for TVs, travelers encounter a walking time cost after parking their cars. For different combinations of travel demands and riding fare of AVs, analytical solutions of three different user equilibrium traffic patterns are obtained. Finally, numerical examples are provided to demonstrate the usefulness of the analytical models. Sensitivity analyses are examined to show the impacts of AV's time-dependent fee and trip-based fixed fee on the traffic pattern and travel costs.

\section{Introduction}

Recent technical progress suggests that autonomous vehicles (AVs) have attracted increasing attention owing to their potential benefits of improving traffic safety, expanding road capacity, saving energy consumption, and reducing vehicular emission [1-3]. With the future deployment of AVs, there would be a stage with heterogeneous traffic streams consisting of both traditional vehicles (TVs) and AVs in road networks. The joint implementation of AV technology and taxi service would bring about a remarkable improvement in travel service. For a taxi company, a taxi fleet with AVs can be more easily operated and its operational efficiency can be substantially enhanced due to the application of information technology in AVs. In turn, travelers can enjoy comfortable AV services and reduction of travel time due to the improvement of traffic flow by reducing random delays [4]. With the implementation of AV-type taxi service, the ownership of private cars would decrease and less land demand would be required for car parking. Researchers also found that AVs can improve freeway traffic flow; for example, Zhou et al. [5] investigated the impact of cooperative AVs in improving freeway merging based on the modified intelligent driver model.

Based on the classical bottleneck model [6-8], we investigate the user equilibrium traffic patterns for a mixed network with both AVs and TVs. Every commuter is assumed to minimize his/her travel cost, including travel time cost, schedule delay penalty, walking time cost for TV drivers, and riding cost for AV passengers, while making decisions on departure time and travel mode. At the equilibrium, no individual can reduce his/her trip travel cost by unilaterally changing his/her departure time and switching travel mode. The first seminal research that characterized the bottleneck congestion effect and departure time choice behavior can be traced back to the 1960s. Vickrey [6] built a single bottleneck rush-hour congestion model for a home-to-work single road network, where all commuters drive to work. Analytical results were derived for the equilibrium traffic pattern. The model proposed by Vickrey [6] is also called 
the bottleneck model, and more detailed extensions can be found in Hendrickson and Kocur [9], Smith [10], Daganzo [11], Cohen [12], Arnott et al. [7, 8], Braid [13], and Zhang et al. [14], to name a few.

The peak-load congestion of multiple routes (modes) with one alternative road bottleneck was also investigated by many researchers, such as Tabuchi [15], Braid [16], Verhoef et al. [17], Yang and Huang [18], Danielis and Marcucci [19], and Huang [20]. Yang and Meng [21] further considered travelers' departure time and route choice behaviors, taking into account the effects of congestion tolls and elastic demand.

One important work considering bottleneck congestion and parking space in a line city is the work of Arnott et al. [7]. They examined the effects of parking on morning rushhour congestion by assuming on-street parking spots spread outside from the CBD (Central Business District). Road tolls and parking fees are used as tools for congestion relief. They found that a spatial parking fee can be used to reverse the parking order and reduce schedule delay cost. Furthermore, the joint implementation of parking fees and road tolls can achieve a system optimum traffic pattern.

In this study, we investigate a user equilibrium traffic pattern based on the traditional bottleneck model considering two travel modes: AVs and TVs. For both TVs and AVs, travelers have to encounter travel costs of queuing delay and schedule delay. However, TVs and AVs have different components of travel costs. Specifically, AV passengers have to pay a riding fare, which comprises a trip-based fixed fee plus a time-dependent variable fee. On the other hand, TV travelers encounter a walking time cost after parking their cars, which is dependent on the parking location.

Arnott et al. [7] showed that TV travelers will depart earlier to compete for a convenient parking spot in order to save walking time. In accordance with this theory, all the $\mathrm{TV}$ travelers depart earlier than the AV travelers for the sake of competing for a parking spot that is closer to the workplace. However, a specific TV or AV traveler can be either early or late for work, depending on the demands of $\mathrm{TV}$ and AV travelers. Therefore, traffic patterns, or departure rates, highly depend on the traffic demands of TV and AV travelers, as well as the model parameters, including schedule delay penalties and fee rates. To analytically investigate the equilibrium traffic pattern, we have to examine the problem in three different scenarios. In the first scenario (scenario A), all AV travelers are assumed to arrive late to work, while TV travelers can arrive either early or late. In the second scenario, all TV travelers are assumed to arrive early to work, while AV travelers can arrive either early or late (scenario B). In the third scenario, both TV and AV travelers can arrive either early or late to work (scenario C).

In the following sections, for different combinations of travel demands and riding fare of AVs, analytical solutions of three different user equilibrium traffic patterns are obtained. Furthermore, numerical examples are provided to demonstrate the usefulness of the analytical models. Sensitivity analyses are also conducted to show the impacts of AV's timedependent fee and trip-based fixed fee on the traffic pattern and travel costs.

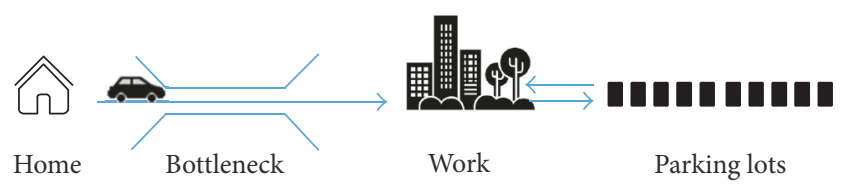

FIGURE 1: An illustrative trip based on the bottleneck model.

\section{The User Equilibrium Traffic Pattern on a Bottleneck Constrained Road}

Figure 1 shows a trip in the morning commuting period, where a traveler departs from home and passes the bottleneck-constrained road by taking either AV or TV and eventually arrives at the workplace, which has a parking pool that includes several parking spots offered at the destination. Suppose that there are $\mathrm{N}$ commuters during the morning commuting period and the travel demand can be further divided between AV users and TV users. It is assumed that the commuters have the same preferred arrival time $t^{*}$ at the workplace, and the fixed service capacity of the bottleneck is set as $s$. Without loss of generality, the free flow travel time on the road is set to zero to facilitate the model analysis.

All TV users have to park their vehicles before they walk to the workplace. Parking spots, which are treated as a continuous variable, are indexed by $n$ in the order of increasing distance outward from the office. Hence, walking time to the office from location $n$ is taken to be $\omega n$, where $\omega$ is the value of unit walking time and it depends on the spatial concentration of parking, walking speed, and so on. Thus, TV users' total travel cost includes the queuing time cost, schedule delay cost, and walking time cost.

Let $\alpha$ be the value of time for TV travelers, and let $\beta$ and $\gamma$ be the penalty costs of early and late arrivals, respectively (empirically $\gamma>\alpha>\beta$ ).

Then, TV users' travel cost is represented by the following function:

$$
\begin{aligned}
C 1= & \alpha(\text { travel time })+\beta(\text { time early })+\gamma(\text { time late }) \\
& +\lambda \cdot(\text { walking time }) .
\end{aligned}
$$

Meanwhile, the travel cost of AV users consists of three parts: a queuing delay cost, a nonpunctual schedule delay cost, and the riding fare, which comprises a trip-based fixed $\operatorname{cost} \tau$ plus a time-dependent variable cost. For a unit time of riding in an AV vehicle, passengers have to pay a timedependent fee $\eta$. Therefore, the cost of AV users is expressed as follows:

$$
\begin{aligned}
C 2= & (\alpha+\eta)(\text { travel time })+\beta(\text { time early }) \\
& +\gamma(\text { time late })+\tau .
\end{aligned}
$$

We set $t^{*}$ as the preferred arrival time, $t_{0}$ as the first user's departure time, and $t_{2}$ as the last user's departure time. In the equilibrium condition, each commuter will face the same travel cost in choosing the departure time; that is, no individual can reduce his/her travel cost by changing his/her departure time. The user equilibrium traffic patterns in the 


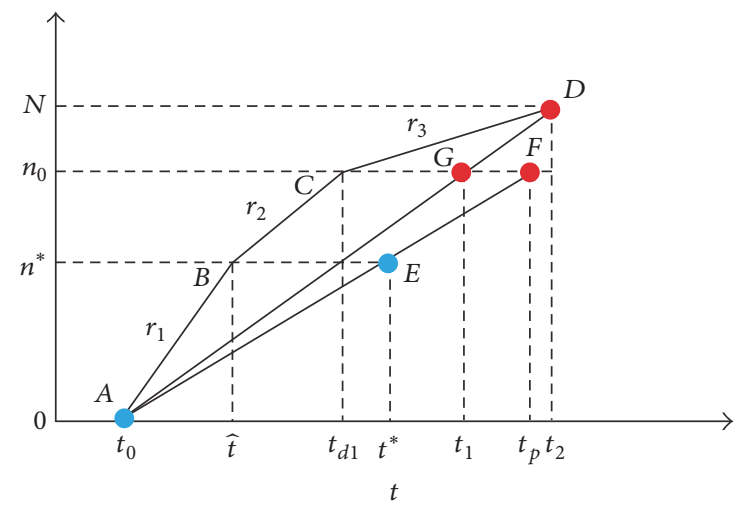

FIGURE 2: User equilibrium traffic pattern for both TVs and AVs in scenario A.

three different scenarios will be discussed in the following section.

2.1. Scenario A: All AV Users Arrive Late. In this scenario, we assume that TV users can arrive early or late, while all $\mathrm{AV}$ users will arrive late. Based on the above assumption, the distribution of users' departure and arrival times is depicted in Figure 2. The first TV user departs at time $t_{0}$. The last TV user departs at time $t_{d 1}$ and arrives at a parking lot at time $t_{1}$. $\mathrm{He} / \mathrm{she}$ then walks to the office and arrives at office at time $t_{p}$. The one who arrives at office on time leaves at $\hat{t}$ and arrives at $t^{*}$, without encountering schedule delay. The last $\mathrm{AV}$ user will leave and arrive at time $t_{2}$. The departure rate for TV users who arrive at office early is $r_{1}$, while that for those who arrive late is $r_{2}$. The departure rate for all AV users who arrive late is $r_{3}$. The total number of TV commuters is $n_{0}$. There are $n^{*}$ commuters who arrive for work before $t^{*}$.

According to the user equilibrium condition, the travel costs of the first and last users should be equal. That is,

$$
\beta\left(t^{*}-t_{0}\right)=\gamma\left(t_{2}-t^{*}\right)+\tau .
$$

All users pass through the bottleneck with full capacity $s$ during the period from $t_{0}$ to $t_{2}$; namely,

$$
t_{2}-t_{0}=\frac{N}{s} \text {. }
$$

Simultaneously solving (3) and (4), the times of the first and last users can be calculated, respectively, as

$$
\begin{aligned}
& t_{0}=t^{*}-\frac{\gamma}{\beta+\gamma} \frac{N}{s}-\frac{\tau}{\beta+\gamma}, \\
& t_{2}=t^{*}+\frac{\beta}{\beta+\gamma} \frac{N}{s}-\frac{\tau}{\beta+\gamma} .
\end{aligned}
$$

The number of cumulative arrivals $n^{*}$ at $t^{*}$ can be expressed as follows:

$$
n^{*}=s\left(t^{*}-\omega n^{*}-t_{0}\right)=\frac{\left(t^{*}-t_{0}\right) s}{1+\omega s} .
$$

The user who arrives on time has no schedule delay cost; thus, his/her total travel cost is denoted as $\left(t^{*}-\omega n^{*}-\widehat{t}\right) \alpha+$ $\lambda \omega n^{*}$, in which $\lambda$ indicates the unit cost for walking time, equaling the first user's total travel cost.

$$
\left(t^{*}-t_{0}\right) \beta=\left(t^{*}-\omega n^{*}-\widehat{t}\right) \alpha+\lambda \omega n^{*}
$$

The departure time $\hat{t}$ of the user who arrives on time is

$$
\widehat{t}=t^{*}-\left(\frac{\beta+(\beta+\alpha-\lambda) \omega s}{(1+\omega s) \alpha}\right)\left(\frac{\gamma}{\beta+\gamma} \frac{N}{s}+\frac{\tau}{\beta+\gamma}\right) .
$$

In equilibrium, $\widehat{t}$ is definitely later than $t_{0}$; we have

$$
\hat{t}>t_{0} \text {. }
$$

Combining (5), (9), and (10), we obtain

$$
\omega<\frac{\alpha-\beta}{(\beta-\lambda) s} .
$$

Similarly, the first AV user and the last AV user have the same travel cost; namely,

$$
(\alpha+\eta)\left(t_{1}-t_{d 1}\right)+\tau+\gamma\left(t_{1}-t^{*}\right)=\gamma\left(t_{2}-t^{*}\right)+\tau .
$$

We can obtain

$$
(\alpha+\gamma+\eta) t_{1}=\gamma t_{2}+(\alpha+\eta) t_{d 1} .
$$

Equating the travel costs of the last TV user and the last $\mathrm{AV}$ user, the following equation is obtained:

$$
\begin{gathered}
\alpha\left(t_{1}-t_{d 1}\right)+\lambda \omega n_{0}+\gamma\left(t_{1}+\omega n_{0}-t^{*}\right) \\
\quad=(\alpha+\eta)\left(t_{1}-t_{d 1}\right)+\tau+\gamma\left(t_{1}-t^{*}\right) .
\end{gathered}
$$

Therefore, we have

$$
[(\gamma+\lambda) \omega s-\eta] t_{1}+\eta t_{d 1}=(\lambda+\gamma) \omega s t_{0}+\tau .
$$

Simultaneously treating (13) and (15), $t_{1}$ and $t_{d 1}$ can be obtained as follows:

$$
\begin{aligned}
t_{1}= & t^{*}-\frac{\tau}{\beta+\gamma}-\frac{r}{\beta+r} \frac{N}{S} \\
& +\frac{r \eta(N / s)+\tau(\alpha+\eta)}{r \eta+(\lambda+\gamma)(\alpha+\eta) \omega s}, \\
t_{d 1}= & t^{*}-\frac{\tau}{\beta+\gamma}-\frac{r}{\beta+r} \frac{N}{S} \\
& +\frac{r \eta(N / s)+\tau(\alpha+\eta)}{r \eta+(\lambda+\gamma)(\alpha+\eta) \omega s} \frac{\alpha+\eta+\gamma}{\alpha+\eta} \\
& -\frac{\gamma}{\alpha+\eta} \frac{N}{S} .
\end{aligned}
$$


The $n_{0}$-th user can choose AV or TV, because both AV and TV have the same travel cost when departing at time $t_{d 1}$, and the slope of arrival curve AG in Figure 2 is $s$. Then,

$$
n_{0}=s\left(t_{p}-t_{0}-\omega n_{0}\right)
$$

It is easy to derive

$$
n_{0}=\frac{s\left(t_{p}-t_{0}\right)}{1+s \omega} .
$$

With $n_{0}=s \cdot t_{1}, t_{p}$ can be expressed by $t_{1}$ and $t_{0}$ as follows:

$$
t_{p}=(1+s \omega) t_{1}-\omega s t_{0}
$$

$$
\begin{aligned}
& \frac{N}{s}<\frac{\tau(\beta+\gamma)}{\gamma \alpha(\gamma+\lambda) \omega s}-\frac{\tau}{\gamma}, \\
& \quad \text { If } \omega>\frac{\beta}{\mathbf{s}(\lambda+\gamma)}, 0<\eta \leq \frac{1}{(\gamma(N / s)+\tau)[\beta-\omega s(\lambda+\gamma)]}\left[\alpha\left[\left(\gamma \frac{N}{s}+\tau\right)(\lambda+\gamma) \omega s-\tau(\beta+\gamma)\right]\right] . \\
& \frac{N}{s}>\frac{\tau(\beta+\gamma)}{\gamma \alpha(\gamma+\lambda) \omega s}-\frac{\tau}{\gamma}, \\
& \quad \text { If } \omega<\frac{\beta}{\mathbf{s}(\lambda+\gamma)}, \eta \geq \frac{1}{(\gamma(N / s)+\tau)[\beta-\omega s(\lambda+\gamma)]}\left[\alpha\left[\left(\gamma \frac{N}{s}+\tau\right)(\lambda+\gamma) \omega s-\tau(\beta+\gamma)\right]\right] . \\
& \frac{N}{s} \leq \frac{\boldsymbol{\beta}(\beta+\gamma)}{\omega s \gamma}-\frac{\tau}{\gamma}, \quad \text { If } \omega=\frac{\beta}{\mathbf{s}(\lambda+\gamma)}, \eta>0, \alpha\left[\left(\gamma \frac{N}{s}+\tau\right)(\lambda+\gamma) \omega s-\tau(\beta+\gamma)\right] \geq 0 .
\end{aligned}
$$

In this scenario, all the AV users will arrive late, and the arrival time $t_{1}$ of the first AV user should be later than the official arrival time $t^{*}$.

$$
t_{1} \geq t^{*}
$$

Combining (16) and (21), we obtain

$$
\begin{aligned}
& \left(\gamma \frac{N}{s}+\tau\right)[\beta-\omega s(\lambda+\gamma)] \eta \\
& \quad \geq \alpha\left[\left(\gamma \frac{N}{s}+\tau\right)(\lambda+\gamma) \omega s-\tau(\beta+\gamma)\right] .
\end{aligned}
$$

The solutions can be obtained for the following situations:
The arrival time $t_{p}$ of the last TV user will not be later than the last AV user's arrival time $t_{2}$.

$$
t_{p} \leq t_{2}
$$

Combining (6) and (20), (24) can be simplified as

$$
[(s \omega+1) \tau-\omega \lambda N] \eta \leq \omega N(\lambda+\gamma) \alpha-(\omega s+1) \tau \alpha .
$$

Because $(s \omega+1) \tau-\omega \lambda N<0$, the range of value $\eta$ that supports the above traffic pattern is given in the following:

$$
\begin{aligned}
\text { If } \quad \tau<\frac{\omega \lambda N}{s \omega+1}, \\
\text { then } \quad \eta>\frac{\omega N(\lambda+\gamma) \alpha-(\omega+1) \tau \alpha}{(s \omega+1) \tau-\omega \lambda N}, \\
\text { if } \tau>\frac{\omega \lambda N}{s \omega+1}, \\
\text { then } \eta<\frac{\omega N(\lambda+\gamma) \alpha-(\omega+1) \tau \alpha}{(s \omega+1) \tau-\omega \lambda N} .
\end{aligned}
$$

Due to user equilibrium, each traveler travel cost is equal, and the total travel cost (TTC) is represented by

$$
\mathrm{TTC}=\left(t^{*}-t_{0}\right) \beta N=\left(\frac{\beta \gamma}{\beta+\gamma} \frac{N}{s}+\frac{\beta \tau}{\beta+\gamma}\right) N .
$$

Furthermore, the total AV company's profit $\left(\mathrm{TAP}_{1}\right)$ in this scenario is

$$
\operatorname{TAP}_{1}=\left(N-n_{0}\right) \tau-\frac{\left(N-n_{0}\right)}{2}\left(t_{1}-t_{d 1}\right) \eta
$$

We can obtain the total system cost $\left(\mathrm{TSC}_{1}\right)$ by subtracting TTC from TAP $_{1}$ :

$$
\begin{aligned}
& \mathrm{TSC}_{1}=\mathrm{TTC}-\mathrm{TAP}_{1}=\left(\frac{\beta \gamma}{\beta+\gamma} \frac{N}{s}+\frac{\beta \tau}{\beta+\gamma}\right) N \\
& -\frac{(N \lambda+N \gamma-\tau) \tau s(\alpha+\eta)}{r \eta+(\lambda+\gamma)(\alpha+\eta) w s} \tau-\frac{\eta}{2} \\
& \frac{(N \lambda+N \gamma-\tau) \tau s(\alpha+\eta)}{r \eta+(\lambda+\gamma)(\alpha+\eta) w s} \\
& \cdot \frac{\gamma}{\alpha+\eta}\left(\frac{N}{s}-\frac{r \eta(N / s)+\tau(\alpha+\eta)}{(\alpha+\eta)(\lambda+\gamma) w s+\eta \gamma}\right) \text {. }
\end{aligned}
$$

2.2. Scenario B: All TV Users Arrive Early at the Destination. Now we turn to the second user equilibrium traffic pattern: all TV users will arrive early, while AV users can arrive either early or late, as shown in Figure 3. In the figure, the parameters, $t_{0}, t_{d 1}, t_{1}, t_{p}, \widehat{t}, t^{*}, n_{0}, n^{*}$, and $t_{2}$ are the same as in Figure 2. The departure rate for those who use TVs and 


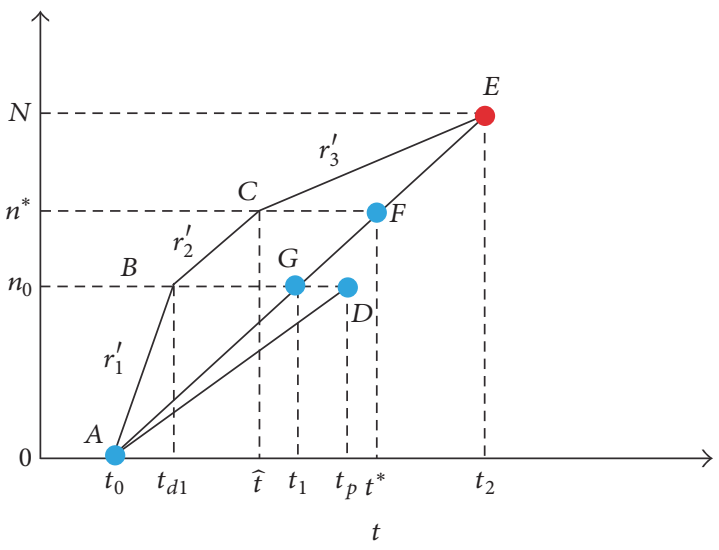

FIgURE 3: User equilibrium traffic pattern for both TVs and AVs in scenario B.

arrive at office early is $r_{1}^{\prime}$, while that for those who arrive early and take AVs is $r_{2}^{\prime}$. The departure rate for AV users who arrive late is $r_{3}^{\prime}$.

Similar to Section 2.1, by equating the schedule delay costs of the first and last arrivals, we can obtain $t_{0}$ and $t_{2}$, which are identical to (5) and (6) in Section 2.1.

In the user equilibrium, equating the travel cost of the first and the last TV users, we have

$$
\alpha\left(t_{1}-t_{d 1}\right)+\lambda \omega n_{0}+\beta\left(t^{*}-t_{p}\right)=\beta\left(t^{*}-t_{0}\right) .
$$

Similarly, the travel cost of the last TV user equals that of the first AV user; namely,

$$
\begin{aligned}
& \alpha\left(t_{1}-t_{d 1}\right)+\lambda \omega n_{0}+\beta\left(t^{*}-t_{p}\right) \\
& \quad=(\alpha+\eta)\left(t_{1}-t_{d 1}\right)+\tau+\beta\left(t^{*}-t_{1}\right) .
\end{aligned}
$$

Simultaneously solving (30) and (31), we obtain the departure time of the last TV user as

$$
\begin{aligned}
t_{d 1}= & t^{*}-\frac{\gamma}{\beta+\gamma} \frac{N}{s}-\frac{\tau}{\beta+\gamma}+\frac{\tau}{\eta} \\
& -\frac{\alpha \tau}{\eta} \frac{\omega s(\lambda-\beta)-\eta}{(\alpha+\eta)(\lambda-\beta) \omega s-\beta \eta}
\end{aligned}
$$

and his/her arrival time as

$$
\begin{aligned}
t_{1}= & t^{*}-\frac{\gamma}{\beta+\gamma} \frac{N}{s}-\frac{\tau}{\beta+\gamma} \\
& +\frac{\alpha \tau}{(\alpha+\eta)(\lambda-\beta) \omega s-\beta \eta} .
\end{aligned}
$$
$t_{0}$ :

Similar to Section 2.1, $t_{p}$ can also be expressed by $t_{1}$ and

$$
t_{p}=t_{1}+\omega n_{0}=(1+s \omega) t_{1}-\omega s t_{0} .
$$

The first TV user's travel cost equals that of the AV user who arrives at office on time.

$$
\beta\left(t^{*}-t_{0}\right)=(\alpha+\eta)\left(t^{*}-\widehat{t}\right)+\tau .
$$

Then,

$$
\widehat{t}=t^{*}+\frac{1}{\alpha+\eta}\left(\tau-\frac{\beta \gamma}{\beta+\gamma} \frac{N}{S}-\frac{\tau \beta}{\beta+\gamma}\right) .
$$

To support the user equilibrium traffic pattern, the following conditions have to be maintained. The last TV user's arrival time $t_{p}$ should be less than the official work start time $t^{*}$; that is, $t_{p}<t^{*}$, which, together with (34), yields

$$
\begin{aligned}
& \frac{\alpha \tau(1+\omega s)}{(\alpha+\eta)(\lambda-\beta) \omega s-\beta \eta}<\frac{\gamma}{\beta+\gamma} \frac{N}{s}+\frac{\tau}{\beta+\gamma}, \\
& \text { If } \omega>\frac{\beta \eta}{s(\alpha+\eta)(\lambda-\beta)}, \\
& \text { then } \eta \\
& \begin{array}{c}
>\frac{\alpha \tau(1+\omega s)(\beta+\gamma)}{\gamma N / S+\tau[(\lambda-\beta) \omega s-\beta]} \\
-\frac{\alpha \omega s(\lambda-\beta)}{\omega s(\lambda-\beta)-\beta}>0 ;
\end{array} \\
& \text { If } \omega<\frac{\beta \eta}{s(\alpha+\eta)(\lambda-\beta)} \text {, } \\
& \text { then } 0<\eta \\
& \begin{aligned}
< & \frac{\alpha \tau(1+\omega s)(\beta+\gamma)}{\gamma N / S+\tau[(\lambda-\beta) \omega s-\beta]} \\
& -\frac{\alpha \omega s(\lambda-\beta)}{\omega s(\lambda-\beta)-\beta} .
\end{aligned}
\end{aligned}
$$

The TTC at the equilibrium in this scenario is the same as that in scenario A, as shown in (27). However, the total AV company's profit in this scenario is different from that in scenario A. We can obtain $\mathrm{TAP}_{2}$ as follows:

$$
\mathrm{TAP}_{2}=\left(N-n^{*}\right) \tau+\frac{N-n^{*}}{2}\left(t^{*}-\widehat{t}\right) \eta .
$$

The total system cost $\left(\mathrm{TSC}_{2}\right)$ is given by

$$
\begin{aligned}
\mathrm{TSC}_{2} & =\mathrm{TTC}-\mathrm{TAP}_{2}=\left(\frac{\beta \gamma}{\beta+\gamma} \frac{N}{s}+\frac{\beta \tau}{\beta+\gamma}\right) N \\
- & \left(\frac{\gamma}{\beta+\gamma} \frac{N}{s}+\frac{\tau}{\beta+\gamma}\right) s \tau \\
- & \frac{\eta}{\alpha+\eta}\left(\frac{\beta \gamma}{\beta+\gamma} \frac{N}{S}+\frac{\tau \beta}{\beta+\gamma}-\tau\right) \\
. & \left(\frac{\gamma}{\beta+\gamma} \frac{N}{s}+\frac{\tau}{\beta+\gamma}\right) \frac{s \tau}{2} .
\end{aligned}
$$

2.3. Scenario C: Both TV and AV Users Can Arrive Early or Late. In this scenario, both TV and AV users can arrive either early or late, where departure rate changes from early arrival to late arrival for both TV and AV travelers, as shown in Figure 4 . The departure rate for TV users who arrive at office 


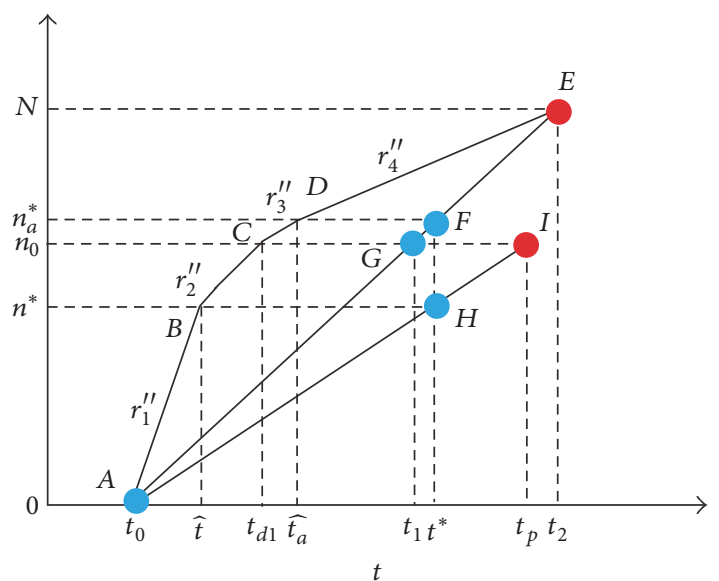

FIgURE 4: User equilibrium traffic pattern for both TVs and AVs in scenario C.

early is $r_{1}^{\prime}$, while the departure rate for TV users who arrive late is $r_{2}^{\prime}$. In the figure, the parameters, $t_{0}, t_{d 1}, t_{1}, t_{p}, t^{*}, n_{0}$, and $t_{2}$ are the same as in Figure 2. Suppose that $\widehat{t}$ is the departure time of the TV traveler who arrives on time and $\widehat{t}_{a}$ is the departure time of the AV traveler who arrives on time. The departure rate for AV users who arrive early is $r_{3}^{\prime}$, while that for AV users who arrive late is $r_{4}^{\prime}$.

In the user equilibrium, the travel cost of the last TV user equals that of the first AV user:

$$
\begin{gathered}
\alpha\left(t_{1}-t_{d 1}\right)+\lambda \omega n_{0}+\gamma\left(t_{1}+\omega n_{0}-t^{*}\right) \\
=(\alpha+\eta)\left(t_{1}-t_{d 1}\right)+\tau+\beta\left(t^{*}-t_{1}\right) .
\end{gathered}
$$

Furthermore, the travel cost of the last AV user equals that of the first AV user:

$$
\tau+\gamma\left(t_{2}-t^{*}\right)=(\alpha+\eta)\left(t_{1}-t_{d 1}\right)+\tau+\beta\left(t^{*}-t_{1}\right) .
$$

Simultaneously solving (40) and (41), we obtain the departure time and arrival time of the last TV user as follows:

$$
\begin{aligned}
t_{1} & =t^{*}+\frac{\tau(\alpha+\eta)+\gamma \eta(N / S)-((\gamma /(\beta+\gamma))(N / s)+\tau /(\beta+\gamma))[(\alpha+\eta)(\lambda+\gamma) \omega s+\eta \gamma]}{(\alpha+\eta)(\lambda+\gamma) \omega s+\alpha \beta+(\alpha+\eta) \gamma}, \\
t_{d 1} & =t^{*} \\
& +\frac{\tau(\alpha+\eta-\beta)-[(\lambda+\gamma) \omega s(\alpha+\eta-\beta-\gamma)+\gamma \eta-\gamma(\beta+\gamma)]((\gamma /(\beta+\gamma))(N / s)+\tau /(\beta+\gamma))-\gamma[(\gamma+\lambda) \omega s+\beta+\gamma-\eta](N / s)}{(\alpha+\eta)(\lambda+\gamma) \omega s+\alpha \beta+(\alpha+\eta) \gamma} .
\end{aligned}
$$

Because the travel cost of the AV user who arrives on time equals that of the last AV user, we have

$$
(\alpha+\eta)\left(t^{*}-\widehat{t}_{a}\right)+\tau=\gamma\left(t_{2}-t^{*}\right)+\tau \text {. }
$$

Then the departure time $\widehat{t}_{a}$ for the AV user who can arrive at the office on time can be obtained as follows:

$$
\widehat{t}_{a}=t^{*}-\frac{((\gamma \beta /(\beta+\gamma))(N / s)-\tau \gamma /(\beta+\gamma))}{(\alpha+\eta)} .
$$

Moreover, similar to Section 2.1, the arrival time of the last TV user is

$$
t_{p}=t_{1}+\omega n_{0}=(1+s \omega) t_{1}-\omega s t_{0}
$$

The user equilibrium traffic pattern requires that the first AV user arrive early for work; that is,

$$
t_{1}<t^{*}
$$

Together with (42), we obtain the following:

$$
\frac{\tau(\alpha+\eta)+\gamma \eta(N / S)-((\gamma /(\beta+\gamma))(N / s)+\tau /(\beta+\gamma))[(\alpha+\eta)(\lambda+\gamma) \omega s+\eta \gamma]}{(\alpha+\eta)(\lambda+\gamma) \omega s+\alpha \beta+(\alpha+\eta) \gamma}<0 .
$$

Therefore, the following inequality about $\eta$ holds:

$$
0<\eta<\frac{\alpha[\tau(\beta+\gamma)-\omega(\lambda+\gamma)(\gamma N+s \tau)]}{[\omega(\lambda+\gamma)(\gamma N+s \tau)-\beta \gamma N / s-\tau(\beta+\gamma)]}
$$

It is also required that the arrival time of the last TV user $t_{p}$ should not be later than the last user's arrival time $t_{2}$ and not be earlier than the punctual arrival time $t^{*}$.

$$
t^{*}<t_{p}<t_{2}
$$

Combining (46) and (50), we obtain

$$
\left[\frac{\gamma}{\beta+\gamma} \frac{N}{s}+\frac{\tau}{\beta+\gamma}\right](\gamma+\lambda) \omega s \alpha-(1+\omega s) \tau \alpha-(\beta+\gamma) \omega s \alpha<\left[(1+\omega s)\left(\tau+\gamma \frac{N}{s}\right)-\left(\frac{\gamma}{\beta+\gamma} \frac{N}{s}+\frac{\tau}{\beta+\gamma}\right)(\lambda+\gamma) \omega s-\left(\frac{\gamma}{\beta+\gamma} \frac{N}{s}+\frac{\tau}{\beta+\gamma}\right) \gamma\right] \eta
$$




$$
\begin{aligned}
& \text { if } \quad \omega>\frac{(\beta+\gamma) \tau}{(\gamma+\lambda) \gamma N+(\lambda-\beta) \tau s-(\beta+\gamma)^{2} s}, \\
& \text { then } \quad \eta>\frac{(1+\omega s)(\tau+\gamma(N / s))-((\gamma /(\beta+\gamma))(N / s)+\tau /(\beta+\gamma))(\lambda+\gamma) \omega s-((\gamma /(\beta+\gamma))(N / s)+\tau /(\beta+\gamma)) \gamma}{[(\gamma /(\beta+\gamma))(N / s)+\tau /(\beta+\gamma)](\gamma+\lambda) \omega s \alpha-(1+\omega s) \tau \alpha-(\beta+\gamma) \omega s \alpha} \\
& \quad>0 ; \\
& \text { if } \quad \omega<\frac{(\beta+\gamma) \tau}{(\gamma+\lambda) \gamma N+(\lambda-\beta) \tau s-(\beta+\gamma)^{2} s}, \\
& \text { then } \quad 0<\eta \\
& \quad<\frac{(1+\omega s)(\tau+\gamma(N / s))-((\gamma /(\beta+\gamma))(N / s)+\tau /(\beta+\gamma))(\lambda+\gamma) \omega s-((\gamma /(\beta+\gamma))(N / s)+\tau /(\beta+\gamma)) \gamma}{[(\gamma /(\beta+\gamma))(N / s)+\tau /(\beta+\gamma)](\gamma+\lambda) \omega s \alpha-(1+\omega s) \tau \alpha-(\beta+\gamma) \omega s \alpha} .
\end{aligned}
$$

Together with (46) and (6), we have

$$
0<\eta<\alpha+\frac{1}{(1+\omega s)(\tau+\gamma(N / s))} \frac{N}{s} .
$$

Considering the inequalities in (49) and (52), it is easy to verify that the following condition is required for supporting the traffic pattern in this scenario.

$$
\begin{gathered}
\frac{\alpha[\tau(\beta+\gamma)-\omega(\lambda+\gamma)(\gamma N+s \tau)]}{[\omega(\lambda+\gamma)(\gamma N+s \tau)-\beta \gamma N / s-\tau(\beta+\gamma)]} \\
<\alpha+\frac{1}{(1+\omega s)(\tau+\gamma(N / s))} \frac{N}{s} .
\end{gathered}
$$

Hence, we obtain the reasonable range of $\eta$ in this scenario as follows:

$$
0<\eta<\frac{\alpha[\tau(\beta+\gamma)-\omega(\lambda+\gamma)(\gamma N+s \tau)]}{[\omega(\lambda+\gamma)(\gamma N+s \tau)-\beta \gamma N / s-\tau(\beta+\gamma)]}
$$

In this scenario, the total AV company's profit is given by

$$
\mathrm{TAP}_{3}=\left(N-n_{a}^{*}\right) \tau-\frac{N-n_{a}^{*}}{2}\left(t^{*}-\widehat{t}_{a}\right) \eta
$$

Furthermore, the total system cost is the total travel cost minus the profit of the AV company:

$$
\begin{aligned}
& \mathrm{TSC}_{3}=\mathrm{TTC}-\mathrm{TAP}_{3}=\left(\frac{\beta \gamma}{\beta+\gamma} \frac{N}{s}+\frac{\beta \tau}{\beta+\gamma}\right) N \\
& -\left(\frac{\gamma}{\beta+\gamma} \frac{N}{s}+\frac{\tau}{\beta+\gamma}\right) s \tau \\
& -\frac{(\gamma \beta /(\beta+\gamma))(N / s)-\tau \gamma /(\beta+\gamma)}{\alpha+\eta}\left(\frac{\gamma}{\beta+\gamma} \frac{N}{s}\right. \\
& \left.+\frac{\tau}{\beta+\gamma}\right) \frac{s \eta}{2} .
\end{aligned}
$$

\section{Numerical Examples}

In this section, three numerical examples are carried out to demonstrate and validate the derivations of the model formulation. We also conduct some numerical analyses to measure the impacts of AVs' trip-based charge and timedependent charge on TSC and TAP and expect to obtain some meaningful insights for traffic management.

3.1. Case I for Scenario A. In this case, all AV commuters arrive late for work, and TV travelers can arrive either early or late at the destination. The home-to-work place network with the following particular conditions is considered: (a) travel demand is set to be 2,000; (b) all the commuters have identical preferred arrival time of 8:00 AM; (c) the bottleneck-constrained road has a service capacity of 500 vehicles per hour and its free flow travel time is set to zero; and (d) other parameters are set as $\alpha=1.0$ Chinese Yuan $(\mathrm{CNY}) / \mathrm{min}, \beta=0.8 \mathrm{CNY} / \mathrm{min}, \gamma=1.0 \mathrm{CNY} / \mathrm{min}, \lambda=$ $1.0 \mathrm{CNY} / \mathrm{min}$, and $\omega=1.0 \mathrm{CNY} / \mathrm{min}$. To arrive at the firsttype user equilibrium state (scenario A), according to the derivations in Section 2.1, the parameter settings of trip-based charge $\tau$ and time-dependent charge $\eta$ for AV users are listed in Table 1.

The computed results in terms of total system cost (TSC) varying with different charge settings are plotted in Figure 5. It can be observed that the TSC increases with the trip-based charge $\tau$, as well as the time-dependent charge. Moreover, it can be found that, given a trip-based charging level, the TSC increases quickly at the beginning and then slows down as the time-dependent charge increases. Normally, when $\eta>5$, TSC remains unchanged. This implies that it would be more effective and sensitive to adjust the trip-based charge rather than the time-dependent charge to pursue a low TSC. That is, under the first-type user equilibrium (scenario A), we suggest setting both trip-based charge and time-dependent charge as low as possible to minimize the TSC.

3.2. Case II for Scenario B. In this case, all TV users arrive early for work, and AV travelers can be either early or late. Here, we set the travel demand as 10,000 and all commuters have the same preferred arrival time of 8:00 AM. The free flow travel time of the bottleneck-constrained road is set to zero and the capacity of the bottleneck is set to be 5,000 vehicles/hour. Other parameters are given as $\alpha$ $=1.0 \mathrm{CNY} / \mathrm{min}, \beta=0.8 \mathrm{CNY} / \mathrm{min}, \gamma=1.2 \mathrm{CNY} / \mathrm{min}, \lambda=$ 
TABLE 1: The setting of trip-based charge $\tau$ and time-dependent charge $\eta$ in Scenario A.

\begin{tabular}{lcc}
\hline Number & $\tau$ & $\eta$ \\
\hline$(1)$ & 0 & {$[0.65,20]$} \\
$(2)$ & 0.1 & {$[0.57,20]$} \\
$(3)$ & 0.2 & {$[0.49,20]$} \\
$(4)$ & 0.3 & {$[0.41,20]$} \\
$(5)$ & 0.4 & {$[0.34,20]$} \\
$(6)$ & 0.5 & {$[0.27,19]$} \\
$(7)$ & 0.6 & {$[0.20,3.3]$} \\
$(8)$ & 0.7 & {$[0.13,1.7]$} \\
$(9)$ & 0.8 & {$[0.07,0.89]$} \\
$(10)$ & 1 & {$[0.00,0.18]$} \\
\hline
\end{tabular}

TABLE 2: The setting of trip-based charge $\tau$ and time-dependent charge $\eta$ in Scenario B.

\begin{tabular}{lcc}
\hline Number & $\tau$ & $\eta$ \\
\hline$(1)$ & 0.01 & {$[0,0.89]$} \\
$(2)$ & 0.02 & {$[0,0.87]$} \\
$(3)$ & 0.04 & {$[0,0.75]$} \\
$(4)$ & 0.06 & {$[0,0.63]$} \\
$(5)$ & 0.08 & {$[0,0.51]$} \\
$(6)$ & 0.1 & {$[0,0.40]$} \\
\hline
\end{tabular}

1.0 $\mathrm{CNY} / \mathrm{min}$, and $\omega=4 \times 10^{-4} \mathrm{CNY} / \mathrm{min}$. To arrive at the second-type user equilibrium state (scenario $\mathrm{B}$ ), according to the derived condition (36), we list the parameter setting of trip-based charge $\tau$ and time-dependent charge $\eta$ for AV users in Table 2.

The results in terms of TSC, TAP, and TAN varying with different charges of $\tau$ and $\eta$ are plotted in Figures 6-8, respectively. Let us first look at the TSC under the secondtype user equilibrium. As shown in Figure 6, we can see some different trends from that appearing in case I. In scenario B, it is shown that TSC increases with the decrease of trip-based charge $\tau$ and decreases with the increase of time-dependent charge $\eta$.

We then look at the variation of total AV profit (TAP). As the trip-based charge $\tau$ increases from 0.01 to 0.1 , the TAP increases steadily. In addition, TAP also increases with the increase of time-dependent charge $\eta$. In this case, increasing either trip-based charge or time-dependent charge is beneficial to the profit of the AV company.

We also analyze the modal split between the two travel alternatives, TV and AV. We here use the total AV numbers (TAN) to characterize the variation of modal split with variable charge levels. Similar to the TSC, the TAN goes down with the increase of the trip-based charge $\tau$ and timedependent charge $\eta$.

In addition, we could determine the optimal charging levels to minimize the TSC and simultaneously maintain a certain level of AV market share. For example, as shown in Table 3, with a market of 9,000 AVs, we can set the tripbased charge of $0.06 \mathrm{CNY}$ and time-dependent charge of $0.25 \mathrm{CNY} / \mathrm{min}$ to obtain the minimal TSC of $9,210 \mathrm{CNY}$.

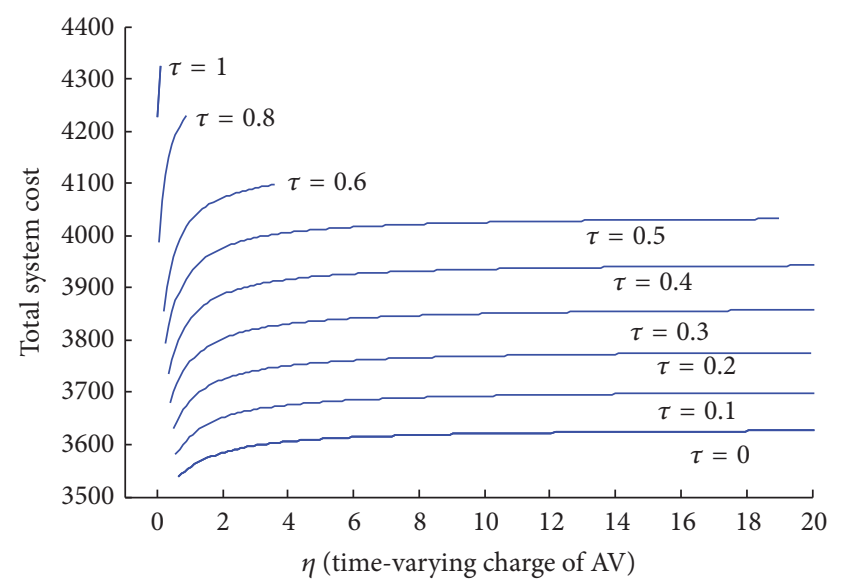

FIGURE 5: TSCs under different levels of trip-based charge $\tau$ and time-dependent charge $\eta$ (case I).

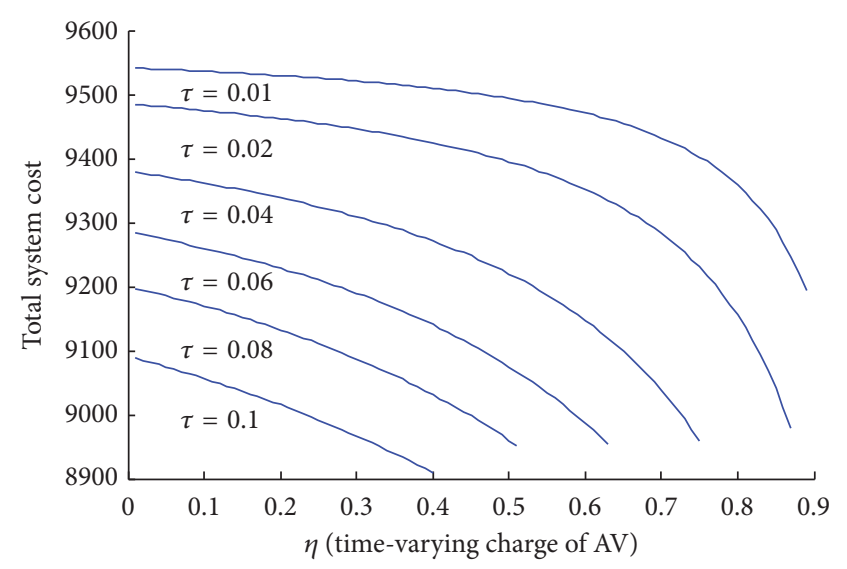

FIgURE 6: TSCs under different levels of trip-based charge $\tau$ and time-dependent charge $\eta$ (case II).

Moreover, when maintaining a market with $8,300 \mathrm{AVs}$, the minimal TSC of 9011 CNY can be obtained by setting the tripbased charge as $0.02 \mathrm{CNY}$ and the time-dependent charge as $0.86 \mathrm{CNY} / \mathrm{min}$. The optimal charging level for the profit of $\mathrm{AV}$ company is different from that of the system optimum in scenario $B$.

3.3. Case III for Scenario C. Recall that, under scenario C, both TV and AV travelers can be early or late for work. The network parameters used in case III are set to be the same as those in case II, expect the parameter $\omega=4 \times 10^{-5} \mathrm{CNY} / \mathrm{min}$. It follows from (51) that if the parameter settings of trip-based charge $\tau$ and time-dependent charge $\eta$ for AV users fall into the ranges given in Table 4, the third-type user equilibrium (scenario C) is obtained.

The TSCs with different charges of $\tau$ and $\eta$ are shown in Figure 9. It can be seen that the TSC increases with the increase of trip-based charge $\tau$ and decreases with the increase of time-dependent charge $\eta$. More specifically, the decreasing rate of the TSC with respect to the time-dependent charge level decreases gradually. Therefore, the network 


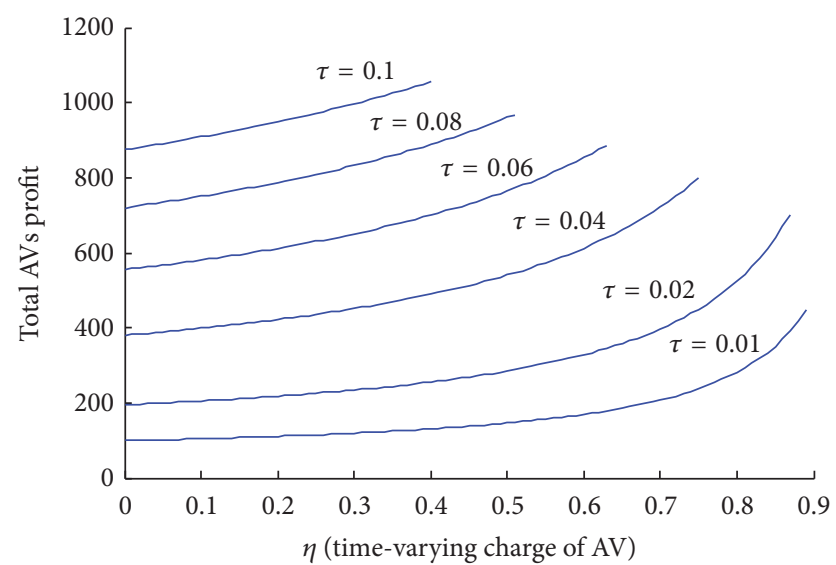

Figure 7: TAPs under different levels of trip-based charge $\tau$ and time-dependent charge $\eta$ (case II).

TABLE 3: The outcomes of TAN and corresponding pricing solutions for case II.

\begin{tabular}{lccc}
\hline TAN $($ Vehs $)$ & $\tau(\mathrm{CNY})$ & $\eta(\mathrm{CNY} / \mathrm{min})$ & TSC $(\mathrm{CNY})$ \\
\hline 9000 & 0.01 & 0.88 & 9221.9 \\
9000 & 0.02 & 0.75 & 9230 \\
9000 & 0.04 & 0.5 & 9220 \\
$\mathbf{9 0 0 0}$ & $\mathbf{0 . 0 6}$ & $\mathbf{0 . 2 5}$ & $\mathbf{9 2 1 0}$ \\
$\mathbf{8 3 0 0}$ & $\mathbf{0 . 0 2}$ & $\mathbf{0 . 8 6}$ & $\mathbf{9 0 1 1 . 1}$ \\
8300 & 0.04 & 0.71 & 9023.7 \\
8300 & 0.06 & 0.56 & 9025.5 \\
8300 & 0.08 & 0.41 & 9024.7 \\
8300 & 0.1 & 0.26 & 9022.9 \\
\hline
\end{tabular}

TABLE 4: The setting of trip-based charge $\tau$ and time-dependent charge $\eta$ in Scenario $C$.

\begin{tabular}{lcc}
\hline Number & $\tau$ & $\eta$ \\
\hline$(1)$ & 0 & {$[0.13,1.22]$} \\
$(2)$ & 0.1 & {$[0.01,0.99]$} \\
$(3)$ & 0.2 & {$[0.01,0.79]$} \\
$(4)$ & 0.3 & {$[0.01,0.60]$} \\
$(5)$ & 0.4 & {$[0.01,0.42]$} \\
$(6)$ & 0.5 & {$[0.01,0.24]$} \\
\hline
\end{tabular}

authority can set a pricing scheme with low trip-based charge and high time-dependent charge to minimize the TSC as much as possible.

Figure 10 shows the TANs varying with different charges of $\tau$ and $\eta$. Obviously, given a trip-based charging level, the TAN decreases with the increase of time-dependent charge $\eta$. For example, when $\tau=0.1$, the TAN is 4695 when $\eta$ is set to zero and the TAN turns to be 3800 when $\eta$ increases to $0.91 \mathrm{CNY} / \mathrm{min}$. The TAN also decreases with the increase of trip-based charge $\tau$. In this scenario, increasing trip-based charge or time-dependent charge will result in the decrease of the TAN in the network.

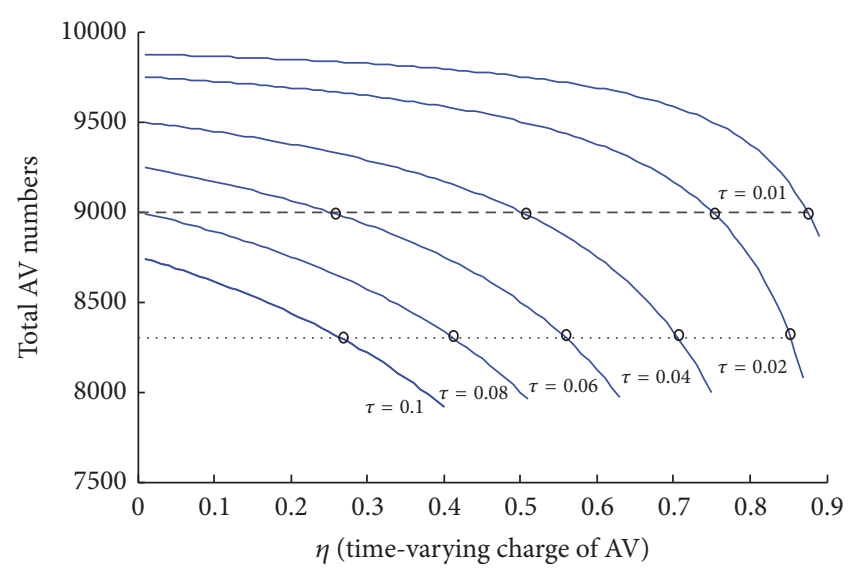

Figure 8: TANs under different levels of trip-based charge $\tau$ and time-dependent charge $\eta$ (case II).

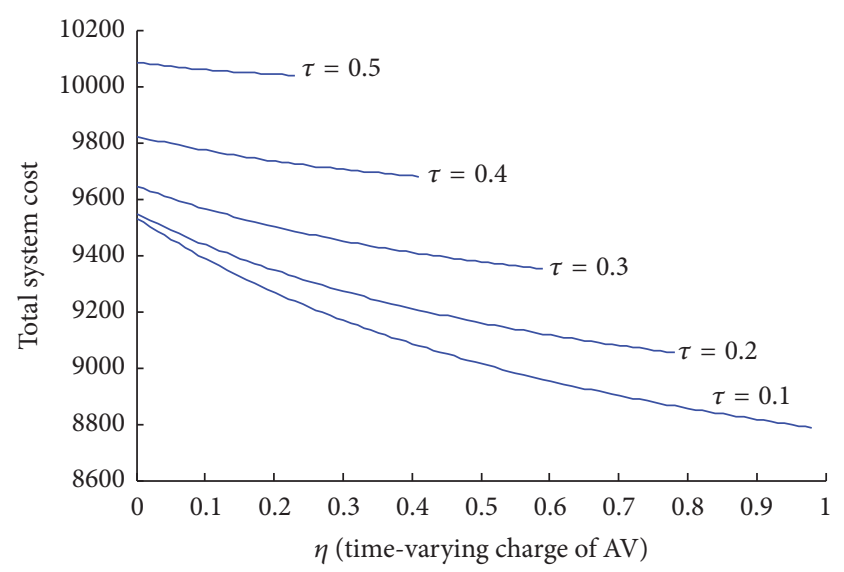

FIGURE 9: TSCs under different levels of trip-based charge $\tau$ and time-dependent charge $\eta$ (case III).

\section{Conclusion}

In this study, the user equilibrium traffic patterns were investigated based on the traditional bottleneck model considering both automatic vehicles (AV) and traditional vehicles (TV). For both TVs and AVs, travelers consider queuing delay and schedule delay as important parts of individual travel cost. On the other hand, they also have different components of travel costs. AV passengers have to pay a riding fare, that is, a trip-based charge plus a riding time-dependent charge; TV travelers encounter a walking time cost after parking their cars at the destination. For different combinations of travel demands and riding fare of AVs, analytical solutions of three different user equilibrium traffic patterns are obtained.

The usefulness of the analytical models is demonstrated in three numerical examples. We investigated the impacts of AV's time-dependent charge rate and the trip-based fixed fee on the TSC, TAP, and TAN. We found that the developed model is helpful in setting a reasonable price for AVs to raise TAP and reduce the TSC. Apart from the above analysis, the model derived in the study can also be further improved for 


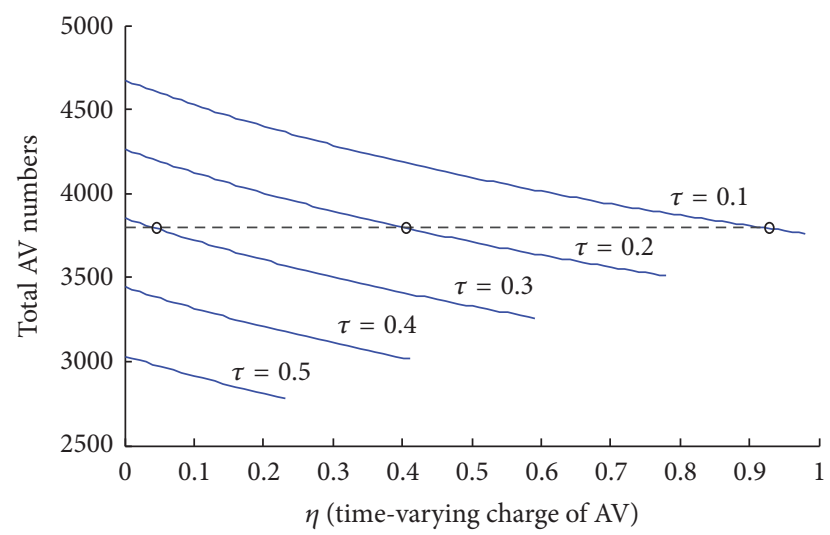

FIGURE 10: TANs under different levels of trip-based charge $\tau$ and time-dependent charge $\eta$ (case III).

application in other related issues, such as setting parking pricing and parking location numbers.

\section{Conflicts of Interest}

The authors declare that there are no conflicts of interest regarding the publication of this paper.

\section{Acknowledgments}

This research was substantially supported by the grants from the National Natural Science Foundation Council of China (71531011 and 71201103), the Humanities and Social Science Project of the Ministry of Education (16YJC630044), Shanghai Soft Science Research Plan Project (17692108800), the Shanghai Pujiang Program (16PJC090), and the Shanghai Shuguang Program (Project no. 13SG23).

\section{References}

[1] S. Shladover, D. Su, and X. Lu, "Impacts of cooperative adaptive cruise control on freeway traffic flow," Transportation Research Record, vol. 2324, pp. 63-70, 2012.

[2] J. B. Greenblatt and S. Saxena, "Autonomous taxis could greatly reduce greenhouse-gas emissions of US light-duty vehicles," Nature Climate Change, vol. 5, no. 9, pp. 860-863, 2015.

[3] A. C. Mersky and C. Samaras, "Fuel economy testing of autonomous vehicles," Transportation Research Part C: Emerging Technologies, vol. 65, pp. 31-48, 2016.

[4] N. Jiang, "Optimal signal design for mixed equilibrium networks with autonomous and regular vehicles," Journal of Advanced Transportation, vol. 2017, pp. 1-13, 2017.

[5] M. Zhou, X. Qu, and S. Jin, "On the impact of cooperative autonomous vehicles in improving freeway merging: a modified intelligent driver model-based approach," IEEE Transactions on Intelligent Transportation Systems, vol. PP, no. 99, pp. 1-7, 2016.

[6] W. Vickrey, "Congestion theory and transport investment," American Economic Review, vol. 59, pp. 251-261, 1969.

[7] R. Arnott, A. de Palma, and R. Lindsey, "A temporal and spatial equilibrium analysis of commuter parking," Journal of Public Economics, vol. 45, no. 3, pp. 301-335, 1991.
[8] R. Arnott, A. de Palma, and R. Lindsey, "A structural model of peak-period congestion: a traffic bottleneck with elastic demand," The American Economic Review, vol. 83, no. 1, pp. 161179, 1993.

[9] C. Hendrickson and G. Kocur, "Schedule delay and departure time decision in a deterministic model," Transportation Science, vol. 15, no. 1, pp. 62-77, 1981.

[10] M. J. Smith, "Existence of a time-dependent equilibrium distribution of arrivals at a single bottleneck," Transportation Science, vol. 18, no. 4, pp. 385-394, 1984.

[11] C. F. Daganzo, "Uniqueness of a time-dependent equilibrium distribution of arrivals at a single bottleneck," Transportation Science, vol. 19, no. 1, pp. 29-37, 1985.

[12] Y. Cohen, "Commuter welfare under peak-period congestion tolls: who gains and who loses?" International Journal of Transport Economics, vol. 14, no. 3, pp. 239-266, 1987.

[13] R. M. Braid, "Peak-load pricing of a transportation route with an unpriced substitute," Journal of Urban Economics, vol. 40, no. 2, pp. 179-197, 1996.

[14] X. Zhang, H.-J. Huang, and H. M. Zhang, "Integrated daily commuting patterns and optimal road tolls and parking fees in a linear city," Transportation Research Part B: Methodological, vol. 42, no. 1, pp. 38-56, 2008.

[15] T. Tabuchi, "Bottleneck congestion and modal split," Journal of Urban Economics, vol. 34, no. 3, pp. 414-431, 1993.

[16] R. M. Braid, "Uniform versus peak-load pricing of a bottleneck with elastic demand," Journal of Urban Economics, vol. 26, no. 3, pp. 320-327, 1989.

[17] E. Verhoef, P. Nijkamp, and P. Rietveld, "Second-best congestion pricing: The case of an untolled alternative," Journal of Urban Economics, vol. 40, no. 3, pp. 279-302, 1996.

[18] H. Yang and H.-J. Huang, "Analysis of the time-varying pricing of a bottleneck with elastic demand using optimal control theory," Transportation Research Part B: Methodological, vol. 31, no. 6, pp. 425-440, 1997.

[19] R. Danielis and E. Marcucci, "Bottleneck road congestion pricing with a competing railroad service," Transportation Research Part E: Logistics and Transportation Review, vol. 38, no. 5, pp. 379-388, 2002.

[20] H.-J. Huang, "Pricing and logit-based mode choice models of a transit and highway system with elastic demand," European Journal of Operational Research, vol. 140, no. 3, pp. 562-570, 2002.

[21] H. Yang and Q. Meng, "Departure time, route choice and congestion toll in a queuing network with elastic demand," Transportation Research Part B: Methodological, vol. 32, no. 4, pp. 247-260, 1998. 


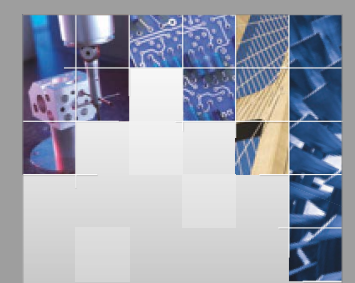

\section{Enfincering}
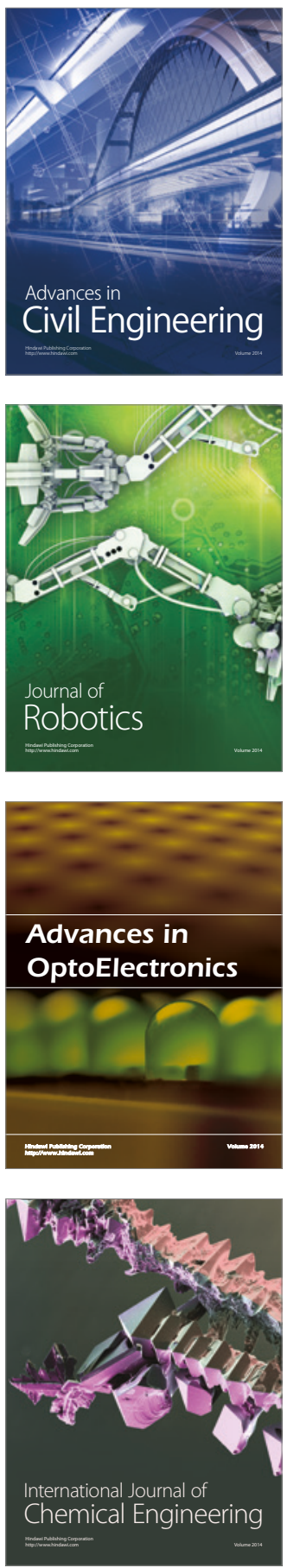

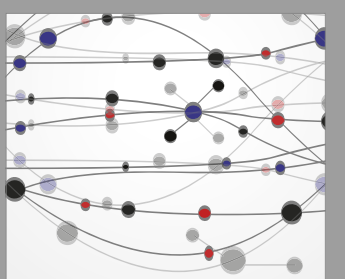

The Scientific World Journal

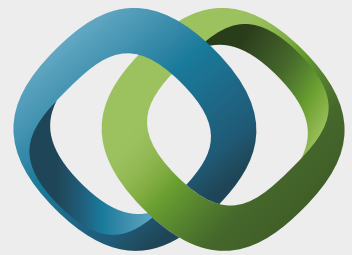

\section{Hindawi}

Submit your manuscripts at

https://www.hindawi.com
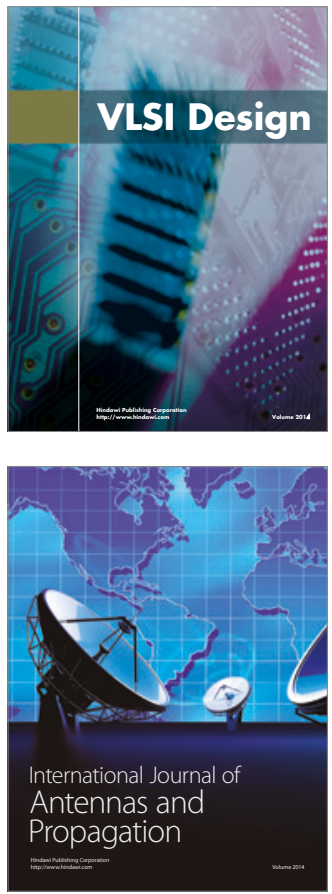

\section{Rotating}

Machinery
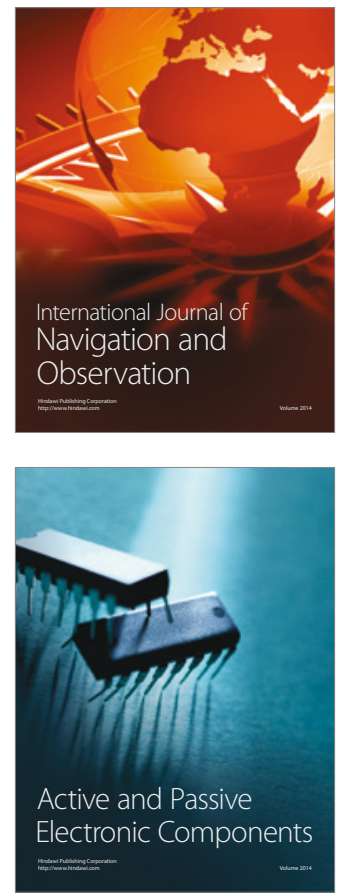
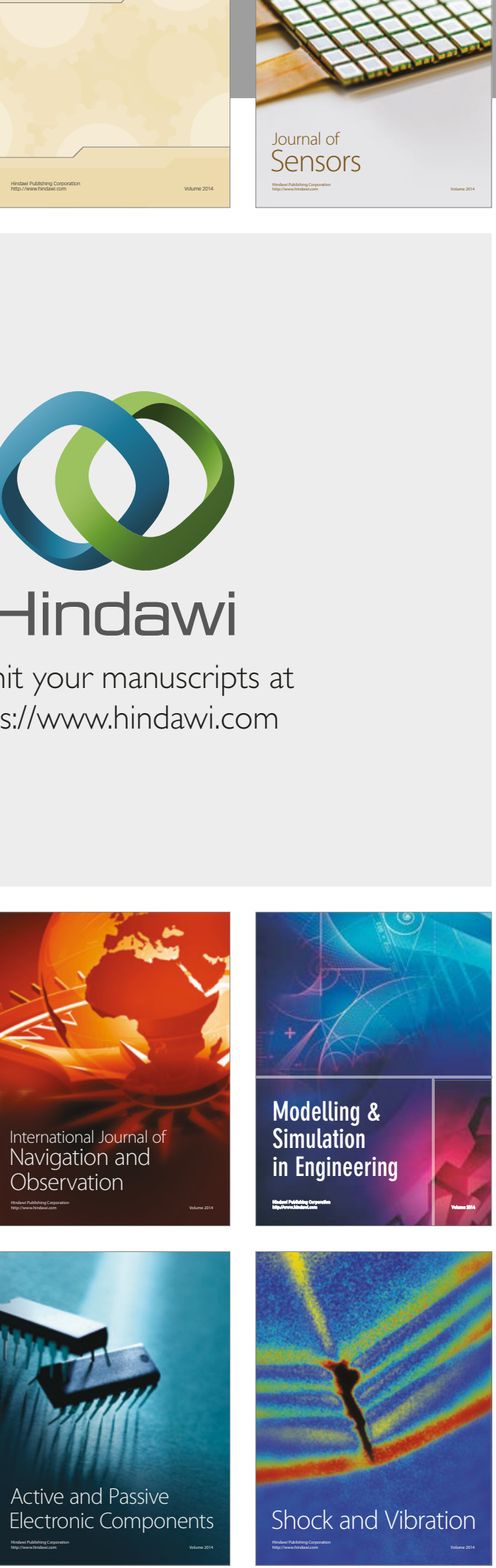
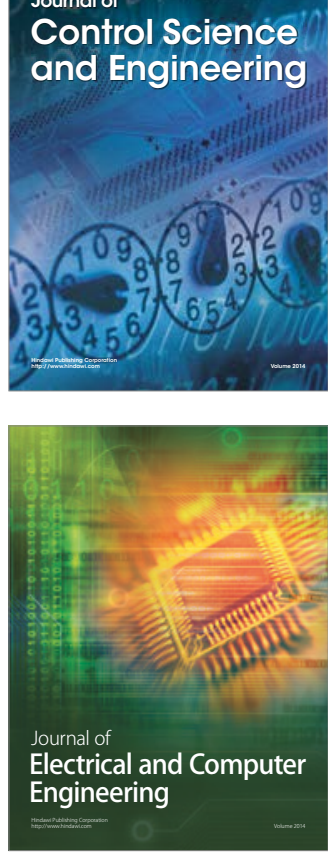

Distributed

Journal of

Control Science

and Engineering
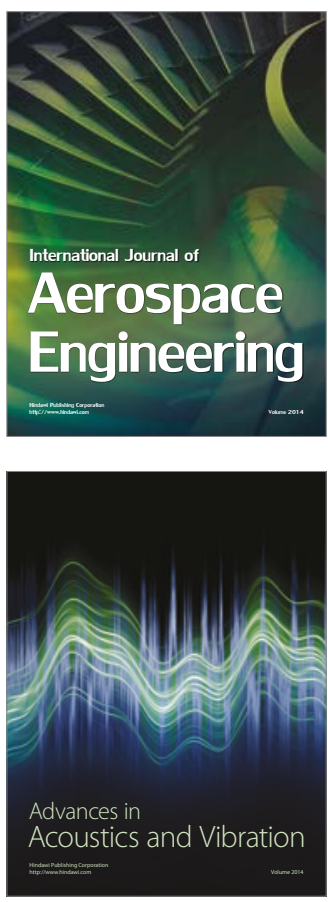

Sensor Networks 